\title{
METHODS AND APPLICATIONS
}

\section{Structural templates for modeling homodimers}

\author{
Petras J. Kundrotas, ${ }^{1}$ Ilya A. Vakser, ${ }^{1 *}$ and Joël Janin ${ }^{2 \star}$ \\ ${ }^{1}$ Center for Bioinformatics, The University of Kansas, 2030 Becker Dr., Lawrence, Kansas 66047 \\ ${ }^{2}$ IBBMC, UMR 8619 CNRS, Université Paris-Sud 11, 91405-Orsay, France
}

Received 21 June 2013; Accepted 23 August 2013

DOI: $10.1002 /$ pro.2361

Published online 31 August 2013 proteinscience.org

\begin{abstract}
Oligomeric proteins are more abundant in nature than monomeric proteins, and involved in all biological processes. In the absence of an experimental structure, their subunits can be modeled from their sequence like monomeric proteins, but reliable procedures to build the oligomeric assembly are scarce. Template-based methods, which start from known protein structures, are commonly applied to model subunits. We present a method to model homodimers that relies on a structural alignment of the subunits, and test it on a set of 511 target structures recently released

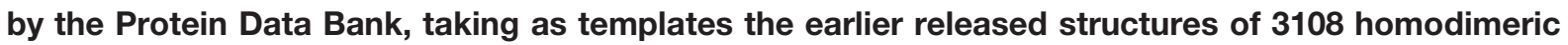
proteins (H-set), and 2691 monomeric proteins that form dimer-like assemblies in crystals (M-set). The structural alignment identifies a $\mathrm{H}$-set template for $97 \%$ of the targets, and in half of the cases, it yields a correct model of the dimer geometry and residue-residue contacts in the target. It also identifies a M-set template for most of the targets, and some of the crystal dimers are very similar to the target homodimers. The procedure efficiently detects homology at low levels of sequence identities, and points to erroneous quaternary structures in the Protein Data Bank. The high coverage of the target set suggests that the content of the Protein Data Bank already approaches the structural diversity of protein assemblies in nature, and that template-based methods should become the choice method for modeling oligomeric as well as monomeric proteins.
\end{abstract}

Keywords: protein modeling; quaternary structure; structural bioinformatics; structure alignment

\section{Introduction}

The great majority of proteins are made of more than one polypeptide chain. Linderström-Lang and Schellman ${ }^{1}$ called their assembly a quaternary structure, individual chains having a primary (the amino acid sequence), a secondary and a tertiary

Grant sponsor: National Institutes of Health; Grant number: R01 GM074255.

*Correspondence to: Joël Janin, IBBMC, UMR 8619 CNRS, Université Paris-Sud 11, Orsay, France. E-mail: joel.janin@upsud.fr or llya A. Vakser, Center for Bioinformatics, The University of Kansas, 2030 Becker Dr., Lawrence, KS 66047. E-mail: vakser@ku.edu (the fold) structure. Monod et al. ${ }^{2}$ coined the word oligomer to describe proteins made of "a few" polypeptide chains. Homo-oligomers comprise chains with identical sequences, the simplest being homodimers. Predicting the three-dimensional structure of a protein from its sequence has been an active field of study in computational biology for over 40 years. $^{3}$ Template-based methods, which search known protein structures to find some on which the query sequence can be modeled, currently dominate the field, thanks in part to the growth of the number of experimentally determined protein structures deposited in the Protein Data Bank (PDB). ${ }^{4}$ Recent 
rounds of the blind prediction experiment CASP (Critical Assessment of Structure Prediction) bear witness to their success. In CASP9 for instance, $80 \%$ of the 129 targets offered for prediction were liable to template-based methods. ${ }^{5,6}$

Quaternary structure has attracted much less interest from computational biologists than tertiary structure, and the submissions made to CASP have almost all been single chain models, even though many of the targets were oligomeric proteins. Macromolecular assemblies may be modeled from smaller structures by "hybrid methods" that take into account constraints derived from electron microscopy, small angle X-ray scattering, mass spectrometry analysis of protein crosslinks, or other experimental data. ${ }^{7-12}$ When the structure of the components is known, at least approximately, model building may proceed by docking components pairwise. Template-free ( $a b$ initio) protein-protein docking is a well-established field, ${ }^{13-17}$ and the performance of docking procedures is assessed by CAPRI (Critical Assessment of PRedicted Interactions), a blind prediction experiment similar to CASP. ${ }^{18,19}$ The method is appropriate for oligomer modeling when an experimental structure of the isolated subunit is available. ${ }^{20}$ But most oligomeric proteins are obligate assemblies, their subunits cannot be studied in isolation, and therefore, they must be model-built before being docked.

Template-based methods apply to docking as well as folding, offering an alternative or a complement to $a b$ initio docking. As docking assumes $a$ priori knowledge of the component structures, templates can be searched for with the help of structural (rather than sequence) alignments. Recent studies indicate that this yields structural templates for nearly all protein-protein hetero-complexes, when their individual components have a known structure. ${ }^{21}$ The method should also be applicable to homo-oligomers. In this study, we use a templatebased docking procedure similar to the one previously developed for modeling of hetero-complexes, ${ }^{21,22}$ to model 511 homodimeric targets of known structure. It relies on the TM-align program ${ }^{23}$ to perform structural alignments of the target subunits on the subunits of other homodimers in the PDB. Those that yield a high score are retained as templates to build the target assembly. The procedure identifies templates for nearly all the homodimers of the test set, and yields a correct model of about half of the targets, often from templates that display a low level of sequence identity to the target, a result similar to our previous study of hetero-complexes. ${ }^{21}$ Homo- and heterodimers are the building blocks of many larger protein assemblies, and likely intermediates in their biogenesis as well as their evolutionary history. ${ }^{2,24-28}$ Higher-order quaternary structures may therefore be amenable to template-based modeling by the stepwise addition of their components, in spite of being more sparsely represented than the dimers in the present PDB.

\section{Results}

\section{The template search}

The targets of this study are 511 homodimeric proteins for which an X-ray structure has been released by the PDB in the year 2011. The templates, which are PDB entries released before 2011, comprise 3108 homodimers (H-set), and 2712 "crystal dimers" formed by crystal symmetries from monomeric proteins (M-set). In all cases, the monomeric or dimeric state is assumed to correspond to the author determined biological unit of the PDB entry.

All structure-based pairwise alignments are generated by applying the TM-align program ${ }^{23}$ to a subunit of the template and of the target. TM-align yields a score (TM-score) in the range 0-1 that quantifies the structural similarity of the subunits; values above 0.5 generally indicate similar folds. ${ }^{29}$ We take the interface C-alpha root-mean-square distance (RMSD) to quantify the similarity of the mode of assembly between the native and the modeled target homodimers. This RMSD, noted IF here, is also used in assessing the quality of CAPRI models. ${ }^{30} \mathrm{~A}$ value of IF below $2 \AA$ means that an accurate model of the target dimer has been built from the template. Values below $5 \AA$ imply that the subunits in the model are similar in position and orientation to the native structure, and correctly reproduce most of the interface residue contacts. The corresponding template is a good starting point for model building, and it should be considered as valid. IF values above $10 \AA$ ascertain that the modes of assembly of the target and the template are unrelated and that only incorrect models can be built from that template. The "gray" area of model accuracy (IF values 5-10 A) approximately corresponds to the docking funnel, ${ }^{31,32}$ which suggests that the models in this zone can be further refined to become near-native. We also evaluate two other parameters used in CAPRI assessment, namely the fraction f_nat of the residue-residue contacts observed in the target that is reproduced (recalled) by the model, and the fraction f_non of the contacts present in the model, but not the target.

\section{H-set templates of homodimeric targets}

The pairwise structural alignments identify at least one putative template with TM-score $>0.5$ in the $\mathrm{H}$-set for all but 15 of the 511 targets, covering 97\% of the target set. With most targets, many $\mathrm{H}$-set entries (35 on average) satisfy that condition, in which case we retain the one that yields the model with the lowest IF and record its rank in the list ordered by decreasing TM-score. This model is 


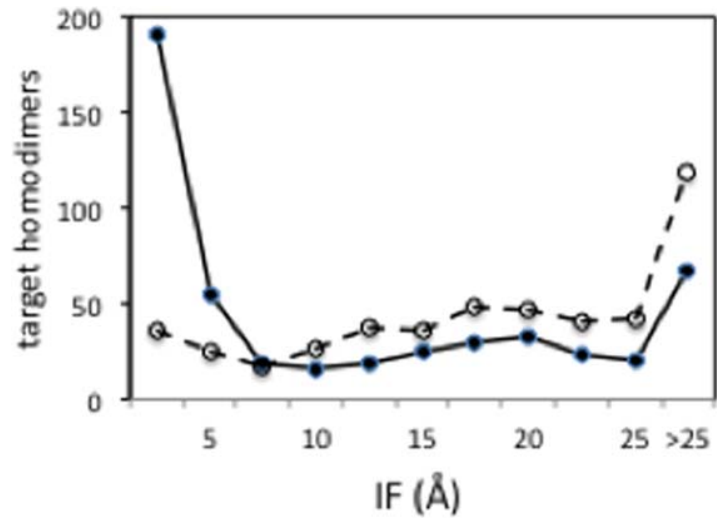

Figure 1. Quality of the best models for the target homodimers. Histogram of IF values representing the root-meansquare distance of the $\mathrm{C}$-alpha atoms of the interface residue in the target native and model structures. The full line is for $\mathrm{H}$-set templates (homodimers), the dashed line for M-set templates (crystal dimers). [Color figure can be viewed in the online issue, which is available at wileyonlinelibrary.com.]

hereafter referred to as the best model, and the corresponding template as the best template. The values of IF, f_nat and f_non cited below refer to that best model.

Figure 1 shows that the distribution of IF for the 496 best models is bimodal. Nearly half have IF $<5 \AA$, which marks the corresponding templates as valid; $44 \%$ have $\mathrm{IF}>10 \AA$, implying that the search yields only false positives (Table I). The remaining $7 \%$ have IF in the 5-10 $\AA$ range, which may include some valid templates along with false positives. When the TM-score cut is raised from 0.5 to 0.6 during the template search, the target coverage reduces to $86 \%$, but the number of best models with IF $<5 \AA$ and the fraction of false positives show little change (from 245 to 240 and $44 \%$ to $40 \%$, respectively).

Of the 496 best templates with TM-score $>0.5$, $36 \%$ yield models with $\mathrm{IF}<2 \AA$, which accurately reproduce the dimer geometry of the target. Figure 2 shows that all these models have f_nat $>0.5$ and f_non $<0.5$. Therefore, a majority of the contacts observed in the target are recalled in the model, and reciprocally. Moreover, the best template in terms of IF also ranks first in terms of TM-score in $75 \%$ of these pairs, in the top ten in $97 \%$.

Another $13 \%$ of the best templates yield models with IF in the range $2-5 \AA$. The great majority still have f_nat $>0.5$ and f_non $<0.5$ (Fig. 2), and therefore, correctly reproduce the residue-residue contacts of the target. These templates are valid, but they do not rank as high in terms of TM-score as those with $\mathrm{IF}<2$ A: only $37 \%$ rank first and $85 \%$ in the top 10.

Some of the models in the IF range 5-10 $\AA$ also recall over half of the native residue-residue contacts, but they display many non-native ones (Fig. 2).
Table I. Sequence and Structure Similarity Between the Target Homodimers and Their Best Templates

\begin{tabular}{|c|c|c|c|c|}
\hline \multirow[t]{2}{*}{ Templates } & \multicolumn{2}{|c|}{$\begin{array}{c}\text { H-set } \\
\text { homodimers }\end{array}$} & \multicolumn{2}{|c|}{$\begin{array}{l}\text { M-set crystal } \\
\text { dimers }\end{array}$} \\
\hline & \multicolumn{2}{|c|}{ Targets (\%) } & \multicolumn{2}{|c|}{ Targets (\%) } \\
\hline All targets & 511 & & 511 & \\
\hline with template & 496 & $97.0^{*}$ & 473 & 92.6 \\
\hline $\mathrm{IF}<2 \AA$ & 180 & 36.3 & 28 & 5.9 \\
\hline $2-5 \AA$ & 65 & 13.1 & 33 & 7.0 \\
\hline $5-10 \AA$ & 35 & 7.0 & 43 & 9.1 \\
\hline $\mathrm{IF}>10 \AA$ & 216 & 43.5 & 368 & 78.0 \\
\hline \multicolumn{5}{|l|}{$\begin{array}{l}\text { Close homologs } \\
\text { (SeqId }>40 \%)\end{array}$} \\
\hline All & 153 & $30.8^{* *}$ & 37 & $7.8^{* *}$ \\
\hline $\mathrm{IF}<2 \AA$ & 133 & 87 & 23 & 62 \\
\hline $2-5 \AA$ & 8 & 5 & 8 & 22 \\
\hline 5-10 & 3 & 20 & 0 & 0 \\
\hline $\mathrm{IF}>10 \AA$ & 9 & 6 & 6 & 16 \\
\hline \multicolumn{5}{|l|}{$\begin{array}{l}\text { Gray zone } \\
\quad(\text { SeqId } 25-40 \%)\end{array}$} \\
\hline All & 63 & $12.7^{* *}$ & 26 & $5.5^{* *}$ \\
\hline $\mathrm{IF}<5 \AA$ & 49 & 78 & 10 & 38 \\
\hline $5-10 \AA$ & 5 & 8 & 3 & 12 \\
\hline $\mathrm{IF}>10 \AA$ & 9 & 14 & 13 & 50 \\
\hline \multicolumn{5}{|l|}{$\begin{array}{l}\text { Unrelated } \\
\qquad \text { (SeqId <25\%) }\end{array}$} \\
\hline All & 280 & $6.5^{* *}$ & 409 & $86.7 * *$ \\
\hline $\mathrm{IF}<5 \AA$ & 55 & 20 & 20 & 5 \\
\hline $5-10 \AA$ & 27 & 10 & 40 & 10 \\
\hline $\mathrm{IF}>10 \AA$ & 198 & 70 & 349 & 85 \\
\hline
\end{tabular}

The percentages marked $\left(^{*}\right)$ refer to the 511 targets, those marked $(* *)$, to the total number of template/target pairs. Other percentages refer to the number of pairs in each SeqId range.

Their templates can be considered as valid only if the non-native contacts can be relieved by refinement without disturbing the overall geometry of the dimer.

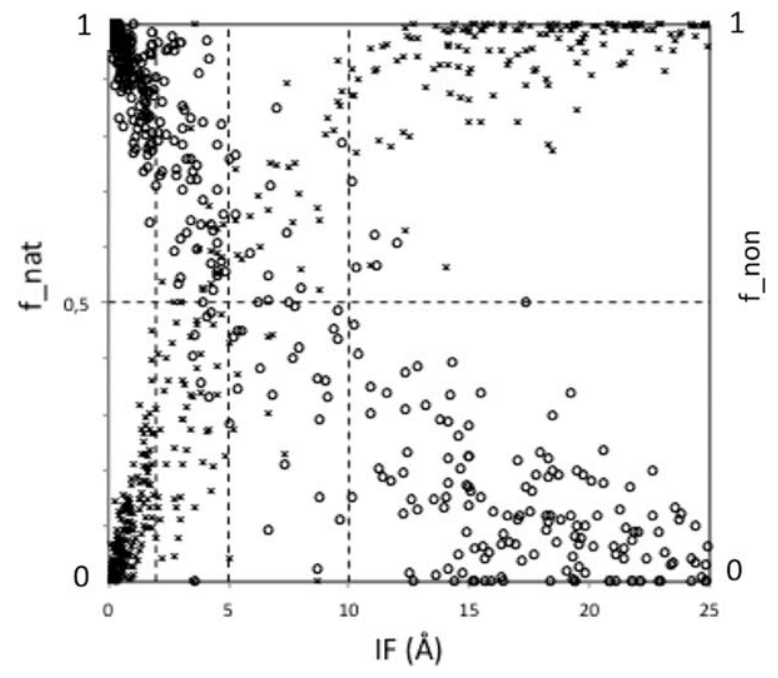

Figure 2. Interface geometry and residue-residue contacts in best models of homodimeric targets built on homodimeric templates. The geometry of the dimer assembly is assessed by IF as in Figure 1; $f$ nat (o) is the fraction of the residue-residue contacts at the interface of the target homodimer that are reproduced in the model; $\mathrm{f} \_$non $(\mathrm{x})$ is the fraction of the residueresidue contacts present in the model, but not the target. 

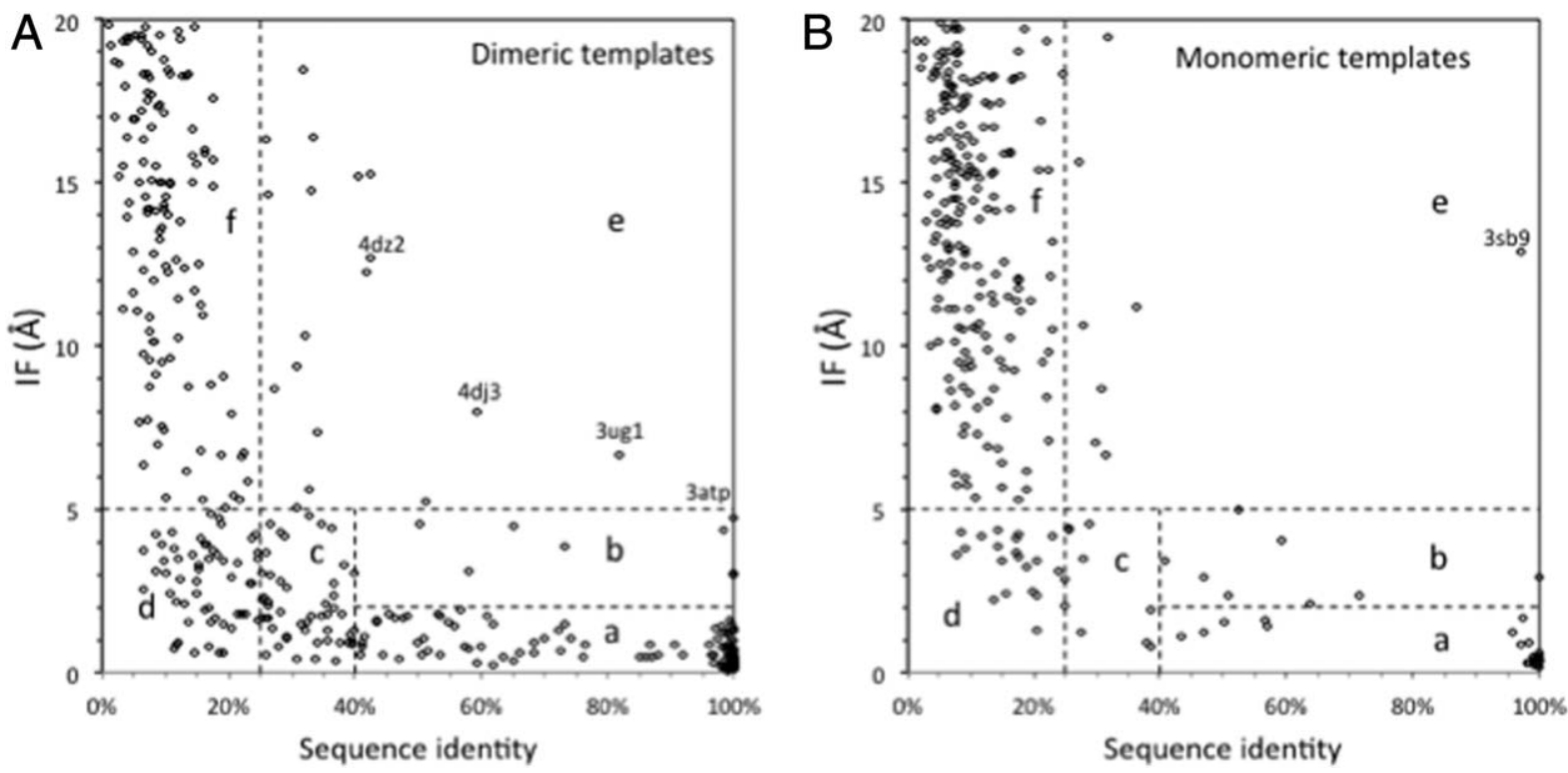

Figure 3. Quality of the best models and sequence identity between the homodimeric targets and their best templates: (A) Hset templates; (B) M-set templates. The IF parameter, which assesses the geometric similarity of the interfaces, is plotted against the percent sequence identity in the target/template pairs identified by the structural alignment search. The plots are divided in six regions as described in the text.

On the other hand, all the models with $\mathrm{IF}>10 \AA$ have f_non $>0.5$; most of the contacts in their interface are non-native, which confirms that these templates are false positives.

\section{Structure versus sequence similarity}

Table I and Figure 3(A) report how the H-set templates identified by the structural alignment are related to the targets in terms of their amino acid sequence. Eighty-six templates/target pairs have identical or nearly identical sequences (SeqId $>97 \%$, allowing for point mutants), and all have $\mathrm{IF}<5 \AA$. About one-third of the templates are close homologs of the targets (SeqId $>40 \%$ ). Another $13 \%$ pairs are in the "gray zone" $(25 \%<$ SeqId $<40 \%)$ where homology is likely, but not certain from the sequence; and a $56.5 \%$ majority displays no detectable homology to the targets (SeqId $<25 \%$ ).

The plot of Figure 3(A) is divided into six regions. Region (a) comprises 133 template/target pairs with SeqId $>40 \%$ and $\mathrm{IF}<2 \AA$, that is, close homologs that form essentially the same dimer assembly. Region (b) comprises 8 pairs in which the template and target are also close homologs, and their dimers have a similar geometry, but display significant differences due to the sequence changes, and/or to allosteric effects, which lead to IF values in the 2-5 $\AA$ range. An allosteric effect explains for instance the change observed between the apo- and ligated states of the bacterial serine chemoreceptor Tsr. $^{33}$ The two allosteric states illustrated by entries 3atp and $2 \mathrm{~d} 4 \mathrm{u}$ have very similar subunit structures (TM-score 0.85), but the dimers differ by IF $=4.7 \AA$, f_nat $=57 \%$, and f_non $=48 \%$.
Region (c) contains 49 template/target pairs that have distantly related sequences (25-40\% SeqId), yet form similar dimers ( $\mathrm{IF}<5 \AA$ ). As the template and target share the same subunit fold and the same mode of dimer assembly, it is very likely that they are homologs, albeit remote. This conclusion can probably be extended to many of the 55 pairs in the region (d) $(\operatorname{SeqId}<25 \%, \mathrm{IF}<5 \AA$ ), but in the absence of a sequence similarity, convergence could be an alternative explanation for the structural similarity expressed by TM-score and IF. All but two pairs in this region are from different organisms, predominantly from bacteria and archaea (36 pairs) and tend to perform the same function (29 pairs). Structural alignments for all but four such pairs cover at least $75 \%$ of the target and/or template structures. Figure 4(A) illustrates the similarity in dimer assembly in Burkholderia pseudomallei nitrilotriacetate monooxygenase (3sdo) and the coenzyme F420 dependent Mycobacterium tuberculosis glucose6-phosphate dehydrogenase FGD1 (3b4y). In spite of the low sequence similarity (SeqId $=16 \%$ ), the structural alignment, which covers $67 \%$ of the target and $92 \%$ of the template and has TM-score 0.81 , indicates that the monomer structures are very similar, especially in the interface region.

Region (d) contains more data points than region (c), yet they make up only $20 \%$ of the pairs with SeqId $<25 \%$, whereas region (c) contains $83 \%$ of those in the gray zone. Region (e) contains 26 pairs in which the template and the target pairs form different dimers although they share the same fold and may be related by their sequence. A few (nine) of the pairs are close homologs, which 

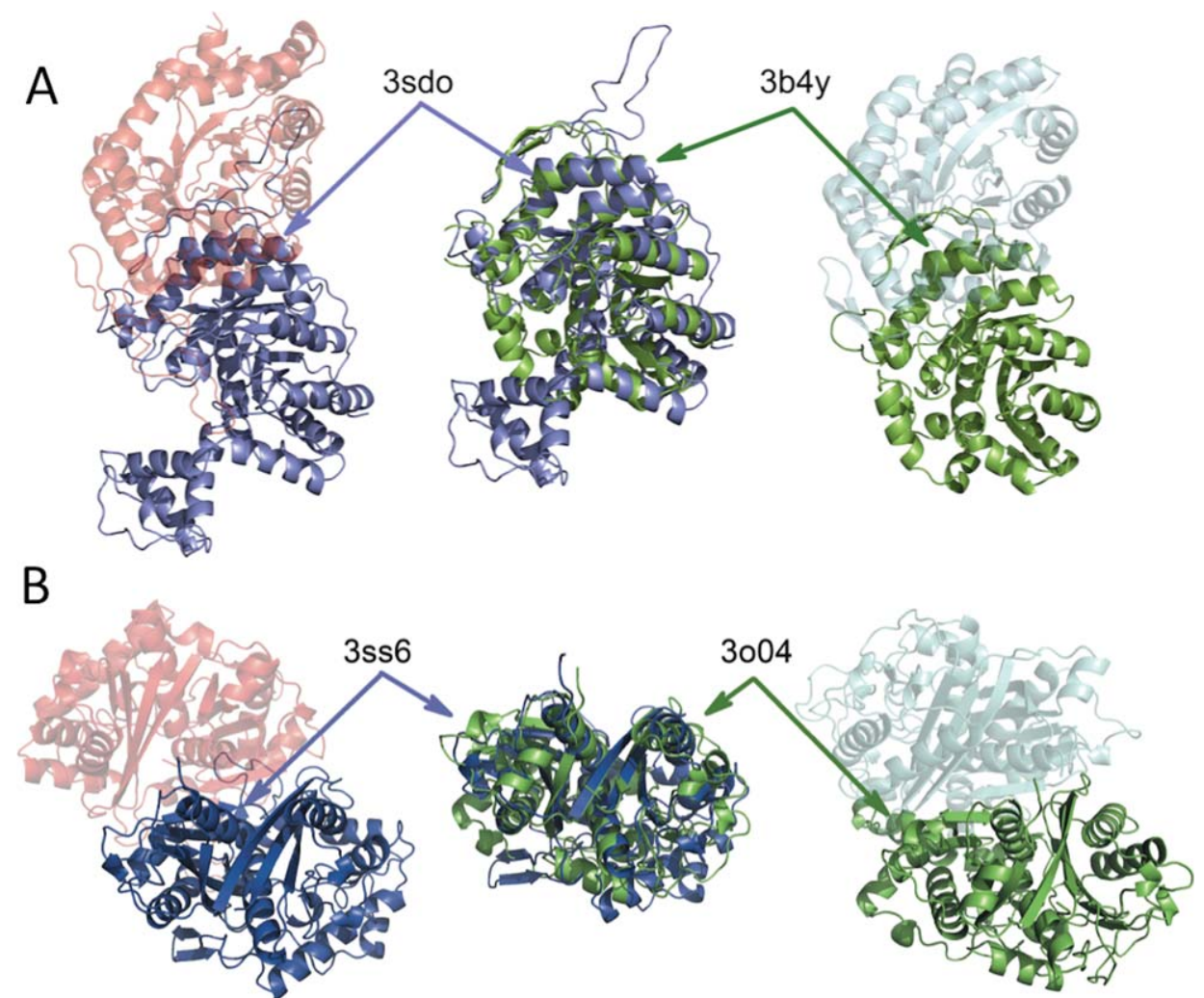

Figure 4. Identifying remote homology and resolving ambiguities in quaternary structure. The left panels represent target homodimers, the right panels, the best templates identified by the search. The central panels show superimposed subunits with the other subunits in the dimers removed for clarity. Arrows point to an almost perfect alignment of the interface $\alpha$-helices. (A) Identifying remote homologies: with Burkholderia pseudomallei nitrilotriacetate monooxygenase (3sdo) as target, the best $\mathrm{H}$-set template is coenzyme F420-dependent Mycobacterium tuberculosis glucose-6-phosphate dehydrogenase FGD1 (3b4y) with Seqld $=16 \%$. A high TM-score $(0.81)$ and similar mode of dimer assembly (IF $=1.9 \AA$, $\left.f \_n a t=0.88, f \_n o n=0.27\right)$ make homology very likely. (B) Resolving ambiguities in quaternary structure: with $B$. anthracis acetyl-CoA acetyl-transferase (3ss6) as target, the best $\mathrm{M}$-set template is a dimer of $L$. monocytogenes beta-keto-acyl carrier protein synthase II (3004) generated by a crystal symmetry, with Seqld $=16 \%$. The quaternary structure reported by the PDB is ambiguous for both proteins: dimeric or tetrameric for $3 s s 6$, monomeric or dimeric for 3004 , but the subunit folds and mode of dimer assemblies are closely related $(\mathrm{TM}$-score $=0.65, \mathrm{IF}=2.4 \AA$, f_nat $=0.85$, f_non $=0.32$ ), and thus, both proteins are likely to be homodimers.

deserves attention. Three are peptidyl-proline isomerases (e.g., 4dz2/3ni6), a protein that binds the drug FK506 at the dimer interface; it is a monomer or forms a different dimer in absence of the drug. ${ }^{34}$ Another pair (3ug1/3gop) contains two different constructs of the kinase domain of the EGF receptor. The template dimer has no two-fold symmetry, ${ }^{35}$ and many of the residues that form its interface are not present in the target construct. Which dimer if any, exists outside the crystal is uncertain. A more convincing example of homologs that dimerize in genuinely different ways is the mouse period circadian protein (4dj3/3gdi), because experiments done in solution and in the cell support the difference seen in crystals of the isoforms present in the template and the target. ${ }^{36}$

Region (f), representing $40 \%$ of all targets, contains pairs in which the template is unrelated to the target in terms of sequence and mode of dimer assembly, indicating that the search yields only false positives. Most of these pairs also have different functions, and the alignments tend to cover a lesser fraction of the subunit structures than for the pairs in region $(d)$.

\section{Monomeric targets and templates}

We also perform alignments of the target homodimer subunits on the M-set proteins reported to be monomeric by their authors in the PDB, and again, find putative templates with TM-score $>0.5$ for most of those targets (93\%, Table I). Conversely, putative templates with TM $>0.5$ are found in the H-set for $95 \%$ of the 859 monomeric targets. Moreover, an average of $29 \mathrm{M}$-set templates per homodimeric target and $32 \mathrm{H}$-set templates per monomeric target satisfy the TM-score $>0.5$ condition, nearly as many as when both targets and templates are homodimers. The great majority of the mixed-state templates are false positives: $78 \%$ of the M-set templates for homodimeric targets (Table I), and 96\% of the H-set templates for monomeric targets, 
yield models with IF $>10 \AA$. This is to be expected if the dimer exists only in the crystal.

Nevertheless, a significant number of the best M-set templates (61 or $13 \%$ ) yields models of homodimeric targets that have IF $<5 \AA$, and 50 of those also have $\mathrm{f}$ nnat $>0.5$ and $\mathrm{f}$ _non $<0.5$. Thus, the crystal dimers these templates form closely resemble the target homodimers. The corresponding target/templates pairs occupy regions (a) to (d) of Figure 3(B), which displays the structural versus sequence similarity in the same way as Figure 3(A). Unexpectedly, 37 of the monomeric templates are identical or nearly identical (SeqId $>97 \%$ ) in sequence to their target. They yield models with IF values below $5 \AA$, with six exceptions. In one exception, the template is ubiquitin and the target is a covalent dimer of ubiquitin (3aul). In another, the template is phage T4 lysozyme, and the target a mutant designed to dimerize in the presence of metal ions (3sb9). ${ }^{37}$ The other four exceptions (3r2v, 3ra5, 3qlm, and 4dz2) are likely to be monomers incorrectly reported to be homodimers by the PDB.

Errors in the opposite direction show up in regions (a) and (b), and possibly in region (c) of Figure $3(\mathrm{~B})$. In these regions, which comprise 41 pairs (9\% of the total), the template is related in sequence to the target, and the crystal dimer closely resembles the target homodimer. It must be a homodimer, not the monomer reported by the PDB. The same conclusion also applies to the 7 (out of 820) monomeric targets that are related in sequence to a $\mathrm{H}$-set template and form a crystal dimer with the same geometry. In such cases, the similarity can help resolving ambiguities in the mode of assembly. Figure 4(B) shows an example: the best template of $B$. anthracis acetyl-CoA acetyltransferase (3ss6) is a dimer of $L$. monocytogenes betaketo-acyl carrier protein synthase II (3004) generated by crystal symmetry. The PDB entries report the first to be a dimer or a tetramer, the second a monomer, or a dimer. The two proteins share a low level of sequence identity, but they have related acyltransferase activities, similar subunit folds, and similar dimer interfaces ( $\mathrm{IF}=2.4 \AA$ ), which makes it likely that both are homodimers.

\section{Discussion}

The PDB released 9847 new protein structures in the period January 2011-April 2012; 45\% are reported to be monomers and $28 \%$, homodimers. PDB statistics (http://www.rcsb.org/pdb/) suggest that homodimers are even more abundant $(48 \%$, against 38\% monomers) in X-ray structures coming from Structural Genomics Centers, which focus on new structures. Indeed, a majority of the targets of the present study are issued from Structural Genomics Centers. These statistics are based on the content of the "biological unit" reported by the PDB as determined by the authors of the structure. This information is not available in all PDB entries, and when it is, it comes with no supporting evidence. The relevant data may not be published, or the publication may disagree with the PDB entry. ${ }^{38,39}$ A common source of error is the confusion between the biological assembly and the content of the asymmetric unit. It is likely to explain why some of our target homodimers can be identical or very close in sequence, to proteins that the PDB entry reports to be monomers even though the same dimer exists in the crystal. In such cases, we checked the publications that describe the structure, and found several that confirm the presence of a homodimer, and belie the "author determined" biological unit of the PDB entry.

The monomer/dimer state of a protein, and in general its quaternary structure, must be determined in solution, which can be a demanding task or yield an ambiguous answer. In a crystal, the noncovalent interactions that stabilize a dimer are of the same physical nature as the crystal contacts, and the difference between the two may be far from obvious. $^{40,41}$ Computational methods have been developed to make the distinction and determine quaternary structures from the data in PDB entries, ${ }^{42-44}$ but their error rate is at least $10 \%$, similar to that of the PDB biological unit. ${ }^{38,45}$ In the present study, a wrongly assigned monomer/dimer state may cause the template search to fail or yield incorrect models, and thus create false negatives. Conversely, finding a valid template implies that the relevant interface is conserved in two different crystal environments, which supports the existence of a homodimer in solution for both the target and the template, although counter-examples do exist. ${ }^{38,39}$

Taking structures released by the PDB in 2011 and early 2012 as targets and older structures as templates, TM-align finds a template with TM-score $>0.5$ for $97 \%$ of the 511 targets. The template is a structural neighbor of the targets, albeit not necessarily a sequence neighbor. The very high coverage of the target set implies that all but a handful of the recently determined homodimer structures have a known fold even when their sequence is novel. The same remark was made for the subunits of the heterodimers, ${ }^{21,46}$ and the result is in line with the CATH database (http://www.cathdb.info), which reports a small increase in the number of superfamilies in recent years: $3 \%$ from 2545 in 2009 to a present 2626.

Another result of the template search is that, in the absence of a sequence similarity, proteins with a similar fold rarely have similar quaternary structures. Given homodimers as targets, the search identifies more templates that have SeqId $<25 \%$ in the $\mathrm{M}$-set than the $\mathrm{H}$-set, and only a small minority (20\%) of the H-set templates yield models similar to the target homodimer. Nevertheless, we find valid 
templates for nearly half of the targets, and most if not all are homologs. Homology is obvious for the 141 template/target pairs that have SeqId $>40 \%$, and almost certain for the 49 of the "gray zone." It is still the most likely explanation of the structural similarity observed in the 55 pairs that have SeqId $<25 \%$ and IF $<5 \AA$. Conversely, most of the templates with SeqId $>25 \%$ are valid and yield good models, but a significant fraction (12\%) does not. Above $40 \%$, a value of IF $>5 \AA$ generally points to an error in the PDB biological unit of either the template or the target. However, the changes that can be observed below $40 \%$ in the dimer geometry or the monomer/dimer state of the two proteins are mostly genuine. This is in line with authors ${ }^{24,47-49}$ who report that while the quaternary structure is almost always conserved between close homologs, changes become frequent for sequence identity below $40 \%$.

Many monomeric proteins are structural neighbors of homodimer subunits. Thus, our procedure may not be able to determine the monomer/dimer state of a protein. But if the target is known to be a homodimer, the search identifies in nearly half of the cases at least one template that ranks among the top 10 in terms of TM-score, and on which a good model of the dimer can be built. This is significantly more than earlier estimates for a sequence comparison. ${ }^{50,51}$ The model accurately reproduces both the dimer geometry and the residue-residue contacts of the target, but it must be recalled that its subunits are taken from the targets, and adopt a conformation appropriate to make a dimer. In an actual prediction, the subunits will be the product of a previous model-building step. Their conformation will not be optimal, and the dimer constructed from the template will need refinement in order to optimize the contacts at the interface.

The present template-based procedure is closely related to one already proposed for modeling heterodimers, which also relies on TM-align to identify structural neighbors of the target subunits. ${ }^{21}$ In each case, the template search achieves a nearly full coverage of test sets made of recently released PDB structures, and it yields a high proportion of valid templates from which good models can be built: $36 \%$ for heterodimers and $49 \%$ for homodimers. When the template is a close homolog of the target, the procedure performs like sequence-based methods, but it can detect homology and build correct models at low levels of sequence identity, where sequencebased methods become unreliable.

The procedure can easily be extended to tetramers, most of which are dimers of dimers, and possibly also to higher oligomers, but those are much fewer in the PDB, and templates may be scarce. It offers an alternative to $a b$ initio docking procedures, a number of which allow building sym- metric oligomers. However, $a b$ initio docking requires the structure of the monomer to be known independently from that of the oligomer, which is very uncommon. Mashiach-Farkash et al. ${ }^{52}$ cite 16 examples, and show that symmetric docking procedures, including their own, yield near-native models of 4-7 of them. A more general approach is the "fold-and-dock" procedure developed by Das et al. ${ }^{53}$ in the Rosetta protein-modeling framework. It alternates steps in which the subunits are modeled de novo by fragment insertion, with steps that perturb the symmetric docking arrangement of the monomers. It was tested on 19 homodimers and 10 other homo-oligomers of known structure, and yielded a correct model of about half. All the test proteins were small, and the largest correct model had only 56 residues per subunit. Das et al.$^{53}$ were also able to "fold-and-dock" some larger proteins for which partial structural information was available from NMR, but their procedure would probably fail or be computationally impractical on most of the homodimers of our test set.

\section{Conclusion}

Protein tertiary structure is highly conserved in evolution, and its conservation can be used to detect homology and build phylogenies at remote stages of divergence. The conservation of the quaternary structure is less stringent, and a monomeric protein often has oligomeric homologs, as in the classical example of myoglobin and hemoglobin. Nevertheless, the present study indicates that, when two proteins are known to be homodimers, a similar tertiary structure generally entails a similar mode of subunit assembly and a conserved geometry of the interface, even though the sequences may be too divergent to be reliably aligned. This observation has implications on the evolution of protein structure and protein-protein interaction, and it extends the range of homology modeling methods to cover many multi-component assemblies of major biological interest.

\section{Methods}

\section{Datasets}

Sets of target and template proteins were generated by extracting protein X-ray structures with a minimum of 50 amino acids per chain from the PDB as of April 13, 2012 (80,740 entries). "Author determined biological units" were taken from REMARK 350 of the PDB entry, ignoring "software determined quaternary structure" information when present. Entries of three types were considered: those where both the asymmetric and the biological unit contain two protein chains with identical sequences (5022 entries); those with one chain in the asymmetric and two in the biological unit (2180 entries); those with 
one chain in both the asymmetric and the biological unit (8844 monomeric entries). Entries without an author determined biological unit, or reporting other oligomeric states than monomer or homodimer, were excluded. Crystal symmetries were applied with the PISA software ${ }^{43}$ to the chain in monomeric entries, and all the pairs of chains that buried at least $250 \AA^{2}$ of interface area per chain were retained, sometimes more than one per entry. This yielded a set of 7417 "crystal dimers" that could be used as targets and templates in the modeling protocol.

The sets were split into targets and templates depending on the release date of the entries: the targets were those released in 2011 and JanuaryApril 2012, the templates, and those released in 2010 and earlier. Redundancy was estimated from the similarity of the structures rather than the sequences after aligning all the chains pairwise with program TM-align, ${ }^{23}$ which produces a measure of structural similarity called TM-score ranging from 0 to $1 .^{29}$ Templates with a TM-score above 0.9 were clustered, and the entry with the best Xray resolution was retained to represent the cluster. Such a high score implies nearly identical folds, and is associated with sequence identity above $40 \%$ in all but a few cases. The threshold value for clustering targets was less stringent, 0.6. Only alignments covering at least $30 \%$ of the smaller protein in the pair were considered during clustering. This ensured that clusters do not contain dissimilar structures in which small structural fragments yield alignments with an artificially high TM-score. This resulted in 511 homodimeric and 859 crystal dimers (monomeric) targets, with the templates being 3108 homodimers (H-set) and 2691 crystal dimers (M-set).

\section{Docking protocol}

The template search was performed by aligning the $\mathrm{C} \alpha$ atoms of a subunit of each target on those of a subunit of either the $\mathrm{H}$-set or the M-set of templates using the TM-align program. The alignment was considered to be statistically significant, and the template retained for further modeling, if the TMscore exceeded 0.5. The same alignment was used to calculate the sequence identity between the target and template.

Noting $\mathrm{A}$ and $\mathrm{A}^{\prime}$ the subunits of the target and $\mathrm{B}$ and $\mathrm{B}^{\prime}$ those of the template, a model of the target homodimer was built by applying to $\mathrm{A}$ the transformation matrix issued from its alignment on $\mathrm{B}$, and to $\mathrm{A}^{\prime}$ the transformation matrix issued from its alignment on $\mathrm{B}^{\prime}$. The quality of this model was evaluated in terms of the root-mean-square distance IF between the interface $\mathrm{C}_{\alpha}$ atoms of subunits $\mathrm{A}^{\prime}$ in the native and the modeled target structures, with subunits A optimally aligned. A residue was considered to be at the interface if any of its atoms was at less than $6 \AA$ of an atom of the other monomer in the target structure. The quantity f_nat was calculated as a fraction of the native interface contacts in the model, and f_non as a fraction of the model interface contacts not present in the native structure.

\section{Acknowledgments}

The authors wish to thank Eugene Krissinel (Chilton, UK) for helping with PISA, and Charles Robert (IBPC, Paris), for discussion.

\section{References}

1. Linderström-Lang KU, Schellman JA (1959) The enzymes. New York: Academic Press, pp 443-510.

2. Monod J, Wyman J, Changeux JP (1965) On the nature of allosteric transitions: a plausible model. J Mol Biol 12:88-118.

3. Dill KA, MacCallum JL (2012) The protein-folding problem, 50 years on. Science 338:1042-1046.

4. Berman H, Henrick K, Nakamura H, Markley JL (2007) The worldwide Protein Data Bank (wwPDB): ensuring a single, uniform archive of PDB data. Nucleic Acids Res 35:D301-D303.

5. Mariani V, Kiefer F, Schmidt T, Haas J, Schwede T (2011) Assessment of template based protein structure predictions in CASP9. Proteins 79:37-58.

6. Moult J, Fidelis K, Kryshtafovych A, Tramontano A (2011) Critical assessment of methods of protein structure prediction (CASP)- Round IX. Proteins 79:1-5.

7. Alber F, Forster F, Korkin D, Topf M, Sali A (2008) Integrating diverse data for structure determination of macromolecular assemblies. Annu Rev Biochem 77: 443-477.

8. Karaca E, Melquiond ASJ, de Vries SJ, Kastritis PL, Bonvin AMJJ (2010) Building macromolecular assemblies by information-driven docking. Mol Cell Proteom 9:1784-1794.

9. Mayne SLN, Patterton HG (2011) Bioinformatics tools for the structural elucidation of multi-subunit protein complexes by mass spectrometric analysis of proteinprotein cross-links. Brief Bioinform 12:660-671.

10. Schneidman-Duhovny D, Hammel M, Sali A (2011) Macromolecular docking restrained by a small angle Xray scattering profile. J Struct Biol 173:461-471.

11. Steven AC, Baumeister W (2008) The future is hybrid. J Struct Biol 163:186-195.

12. Velazquez-Muriel J, Lasker K, Russel D, Phillips J, Webb BM, Schneidman-Duhovny D, Sali A (2012) Assembly of macromolecular complexes by satisfaction of spatial restraints from electron microscopy images. Proc Natl Acad Sci U S A 109:18821-18826.

13. Andrusier N, Mashiach E, Nussinov R, Wolfson HJ (2008) Principles of flexible protein-protein docking. Proteins 73:271-289.

14. Ritchie DW (2008) Recent progress and future directions in protein-protein docking. Curr Protein Pept Sci 9:1-15.

15. Vajda S, Kozakov D (2009) Convergence and combination of methods in protein-protein docking. Curr Opin Struc Biol 19:164-170.

16. Vakser IA, Kundrotas P (2008) Predicting 3D structures of protein-protein complexes. Curr Pharm Biotechno 9:57-66.

17. Wodak SJ, Janin J (2003) Structural basis of macromolecular recognition. Adv Prot Chem 61:9-73. 
18. Janin J (2010) Protein-protein docking tested in blind predictions: the CAPRI experiment. Mol Biosyst 6: 2351-2362.

19. Lensink MF, Wodak SJ (2010) Docking and scoring protein interactions: CAPRI 2009. Proteins 78:30733084.

20. Schneidman-Duhovny D, Inbar Y, Nussinov R, Wolfson HJ (2005) PatchDock and SymmDock: servers for rigid and symmetric docking. Nucleic Acids Res 33:W363W367.

21. Kundrotas PJ, Zhu ZW, Janin J, Vakser IA (2012) Templates are available to model nearly all complexes of structurally characterized proteins. Proc Natl Acad Sci U S A 109:9438-9441.

22. Sinha R, Kundrotas PJ, Vakser IA (2010) Docking by structural similarity at protein-protein interfaces. Proteins 78:3235-3241.

23. Zhang Y, Skolnick J (2005) TM-align: a protein structure alignment algorithm based on the TM-score. Nucleic Acids Res 33:2302-2309.

24. Dayhoff JE, Shoemaker BA, Bryant SH, Panchenko AR (2010) Evolution of protein binding modes in homooligomers. J Mol Biol 395:860-870.

25. Jaenicke R, Lilie H (2000) Folding and association of oligomeric and multimeric proteins. Adv Prot Chem 53 53:329-401.

26. Perica T, Chothia C, Teichmann SA (2012) Evolution of oligomeric state through geometric coupling of protein interfaces. Proc Natl Acad Sci U S A 109:8127-8132.

27. Perica T, Marsh JA, Sousa FL, Natan E, Colwell LJ, Ahnert SE, Teichmann SA (2012) The emergence of protein complexes: quaternary structure, dynamics and allostery. Biochem Soc T 40:475-491.

28. Venkatakrishnan AJ, Levy ED, Teichmann SA (2010) Homomeric protein complexes: evolution and assembly. Biochem Soc T 38:879-882.

29. Zhang Y, Skolnick J (2004) Scoring function for automated assessment of protein structure template quality. Proteins 57:702-710.

30. Lensink MF, Mendez R, Wodak SJ (2007) Docking and scoring protein complexes: CAPRI 3rd edition. Proteins 69:704-718.

31. Hunjan J, Tovchigrechko A, Gao Y, Vakser IA (2008) The size of the intermolecular energy funnel in protein-protein interactions. Proteins 72:344-352.

32. Tovchigrechko A, Wells CA, Vakser IA (2002) Docking of protein models. Prot Sci 11:1888-1896.

33. Tajima H, Imada K, Sakuma M, Hattori F, Nara T, Kamo N, Homma M, Kawagishi I (2011) Ligand specificity determined by differentially arranged common ligand-binding residues in bacterial amino acid chemoreceptors Tsr and Tar. J Biol Chem 286:42200-42210.

34. Vanduyne GD, Standaert RF, Karplus PA, Schreiber SL, Clardy J (1991) Atomic-structure of Fkbp-Fk506, an immunophilin-immunosuppressant complex. Science 252:839-842.

35. Brewer MR, Choi SH, Alvarado D, Moravcevic K, Pozzi A, Lemmon MA, Carpenter G (2009) The juxtamembrane region of the EGF receptor functions as an activation domain. Mol Cell 34:641-651.
36. Kucera N, Schmalen I, Hennig S, Ollinger R, Strauss HM, Grudziecki A, Wieczorek C, Kramer A, Wolf E (2012) Unwinding the differences of the mammalian PERIOD clock proteins from crystal structure to cellular function. Proc Natl Acad Sci U S A 109:3311-3316.

37. Laganowsky A, Zhao ML, Soriaga AB, Sawaya MR, Cascio D, Yeates TO (2011) An approach to crystallizing proteins by metal-mediated synthetic symmetrization. Protein Sci 20:1876-1890.

38. Xu QF, Canutescu AA, Wang GL, Shapovalov M, Obradovic Z, Dunbrack RL (2008) Statistical analysis of interface similarity in crystals of homologous proteins. J Mol Biol 381:487-507.

39. Xu QF, Dunbrack RL (2011) The protein common interface database (ProtCID)-a comprehensive database of interactions of homologous proteins in multiple crystal forms. Nucleic Acids Res 39:D761-D770.

40. Janin J, Bahadur RP, Chakrabarti P (2008) Proteinprotein interaction and quaternary structure. Q Rev Biophys 41:133-180.

41. Janin J, Rodier F, Chakrabarti P, Bahadur RP (2007) Macromolecular recognition in the Protein Data Bank. Acta Cryst D63:1-8.

42. Duarte JM, Srebniak A, Scharer MA, Capitani G (2012) Protein interface classification by evolutionary analysis. BMC Bioinformatics 13:334.

43. Krissinel E, Henrick K (2007) Inference of macromolecular assemblies from crystalline state. J Mol Biol 372: 774-797.

44. Zhu HB, Domingues FS, Sommer I, Lengauer T (2006) NOXclass: prediction of protein-protein interaction types. BMC Bioinformatics 7:27.

45. Levy ED (2007) PiQSi: protein quaternary structure investigation. Structure 15:1364-1367.

46. Zhang QC, Petrey D, Norel R, Honig BH (2010) Protein interface conservation across structure space. Proc Natl Acad Sci U S A 107:10896-10901.

47. Aloy P, Ceulemans H, Stark A, Russell RB (2003) The relationship between sequence and interaction divergence in proteins. J Mol Biol 332:989-998.

48. Andreani J, Faure G, Guerois R (2012) Versatility and invariance in the evolution of homologous heteromeric interfaces. Plos Comput Biol 8:e10026677.

49. Levy ED, Erba EB, Robinson CV, Teichmann SA (2008) Assembly reflects evolution of protein complexes. Nature 453:U1262-U1266.

50. Kundrotas P, Lensink MF, Alexov E (2008) Homologybased modeling of 3D structures of protein-protein complexes using alignments of modified sequence profiles. Int J Biol Macromol 43:198-208.

51. Kundrotas PJ, Zhu ZW, Vakser IA (2010) GWIDD: genome-wide protein docking database. Nucleic Acids Res 38:D513-D517.

52. Mashiach-Farkash E, Nussinov R, Wolfson HJ (2011) SymmRef: a flexible refinement method for symmetric multimers. Proteins 79:2607-2623.

53. Das R, Andre I, Shen Y, Wu YB, Lemak A, Bansal S, Arrowsmith CH, Szyperski T, Baker D (2009) Simultaneous prediction of protein folding and docking at high resolution. Proc Natl Acad Sci U S A 106:18978-18983. 